\title{
SOLUTION OF THE MULTIDIMENSIONAL DIFFUSION EQUATION USING LIE SYMMETRIES: SIMULATION OF POLLUTANT DISPERSION IN THE ATMOSPHERE
}

\author{
J. R. Zabadala, \\ and C. A. Poffal ${ }^{\mathrm{b}}$

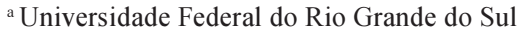 \\ Departamento de Engenharia Nuclear \\ Porto Alegre, Rio Grande do Sul, 90.046-900, \\ Brazil \\ jorge.zabadal@ufrgs.br \\ ${ }^{\mathrm{b}}$ Universidade Federal do Rio Grande do Sul \\ Programa de Pós-graduação em Engenharia \\ Mecânica \\ Porto Alegre, Rio Grande do Sul, 90.046-900, \\ Brazil \\ poffal@brturbo.com.br
}

\begin{abstract}
Several analytical, numerical and hybrid methods are being used to solve diffusion and diffusion advection problems. In this work, a closed form solution of the three-dimensional diffusion advection equation in a Cartesian coordinate system is obtained by applying rules, based on the Lie symmetries, to manipulate the exponential of the differential operators that appear in its formal solution. There are many advantages of applying these rules: the increase in processing velocity so that the solution may be obtained in real time, the reduction in the amount of memory required to perform the necessary tasks in order to obtain the solution, since the analytical expressions can be easily manipulated in postprocessing and also the discretization of the domain may not be necessary in some cases avoiding the use of mean values for some parameters involved. These rules yield good results when applied to obtain solutions for problems in fluid mechanics and in quantum mechanics. In order to show the performance of the method, a one-dimensional scenario of the pollutant dispersion in a stable boundary layer is simulated, considering that the horizontal component of the velocity field is dominant and constant, disregarding the other components. The results are compared with data available in the literature.
\end{abstract}

Keywords: diffusion advection equation, pollutant dispersion, Lie symmetries

\section{NOMENCLATURE}

c pollutant concentration, $\mathrm{Kg} / \mathrm{m}^{3}$

$h$ boundary layer height, $\mathrm{m}$

$h_{s} \quad$ source height, $\mathrm{m}$

I, identity operator

$K_{x} \quad$ longitudinal diffusion coefficient, $\mathrm{m}^{2} / \mathrm{s}$

$K_{y} \quad$ lateral diffusion coefficient, $\mathrm{m}^{2} / \mathrm{s}$

$K_{z} \quad$ vertical diffusion coefficient, $\mathrm{m}^{2} / \mathrm{s}$

$L \quad$ Monin-Obukov length, $\mathrm{m}$

$Q \quad$ instantaneous source, $\mathrm{g} / \mathrm{m}^{2}$

$S \quad$ pollutant source, $\mathrm{Kg} / \mathrm{m}^{3}$

$t$ period of time since the emission, $\mathrm{s}$

$u \quad$ mean wind component in $\mathrm{x}$ direction, $\mathrm{m} / \mathrm{s}$

$u_{*} \quad$ friction velocity, $\mathrm{m} / \mathrm{s}$

$v$

\section{Greek symbols}

$\alpha_{1}$ experimental constant used to determine the Monin-Obukov length $\alpha_{2} \quad$ experimental constant used to determine the Monin-Obukov length

$\delta(x) \quad$ generalized Dirac function

$\Lambda \quad$ local Monin-Obukov length

\section{INTRODUCTION}

In the last decades, interest in studying pollutant dispersion is increasing considerably as a consequence of the environmental problems caused by industrial development. Several analytical, numerical and hybrid methods are used to solve diffusion problems and advection diffusion problems (Zwillinger, 1997).

An exact solution for the general form of the three-dimensional advection diffusion equation (Eq. 1) is not yet known.

$$
\begin{aligned}
& \frac{\partial c}{\partial t}+u \frac{\partial c}{\partial x}+v \frac{\partial c}{\partial y}+w \frac{\partial c}{\partial z} \\
& =\frac{\partial}{\partial x}\left(K_{x} \frac{\partial c}{\partial x}\right)+\frac{\partial}{\partial y}\left(K_{y} \frac{\partial c}{\partial y}\right)+\frac{\partial}{\partial z}\left(K_{z} \frac{\partial c}{\partial z}\right)+S
\end{aligned}
$$

where $c$ represents the pollutant concentration, $t$ is the period of time since the pollutant emission, $K_{x}, K_{y}$ and $K_{z}$ are the longitudinal, lateral and vertical diffusion coefficients, respectively, $u, v$ 
and $w$ correspond to the mean wind components in $x, y$ and $z$ directions, respectively, and $S$ is the source term.

Nieuwstadt (Nieuwstadt, 1980) obtained a solution to the transient one-dimensional problems considering the velocity $u$ constant and taking $K_{z}$ as a function of the height and of the friction velocity $u_{*}$ employing Legendre polynomials. Koch (1989) presented an analytical solution to the two-dimensional problem for a source on the ground, considering power type profiles for the wind and diffusion coefficients, and also the pollutant absorption effect by the soil. Moura (1995) proposed an analytical solution to the turbulent vertical dispersion of pollutants in a stable boundary layer applying Laplace transform, the dispersion coefficient was considered as a function of the height of the stable boundary layer proposed by Degrazia and Moraes (1992). Pires (1996) obtained a similar solution for a convective boundary layer. Applying the same method, Moreira (1996) proposed a solution for the steady-state two-dimensional dispersion problem in a convective boundary layer. Chrysikopoulous et al. (1992) solved a threedimensional problem for a continuous source on ground level, considering that wind velocity and the diffusivities have power type profiles. Moura (1999) proposed an analytical solution for the steady-state two and three-dimensional equations using the generalized integral transform. The solutions were valid when the pollutant was subjected to homogenous turbulence and when the mean values of the wind velocity components were uniform. Moreira (2000) presented an analytical model of dispersion based on discretization of the planetary boundary layer. In each sub-layer the advection diffusion equation was solved by means of the application of Laplace transform, considering mean values for the vertical diffusion coefficient and for the wind velocity.

The aim of this paper is to present an analytical formulation to determine the solution for the advection diffusion equation in a Cartesian coordinate system. This formulation consists in the application of the rules, based on Lie symmetries, for manipulation of exponentials of differential operators appearing in the formal solution. In order to test the performance of the method, a one-dimensional scenario of pollutant dispersion in a stable boundary layer is simulated.

The application of the rules for manipulation of the exponentials of differential operators makes the execution possible in a usual $\mathrm{PC}$, reducing the amount of memory required and increasing the processing speed, alolowing the solution to be obtained in real time. The method is efficient to solve problems in quantum (Zabadal et al., 2001) and fluid mechanics (Zabadal et al., 2004) and to simulate pollutant dispersion in rivers.

\section{Rule for Manipulation of the Exponential of a First Order Differential Operator}

In 1870 Marius Sophus Lie found out that methods used to solve separable, homogenous and exact differential equations are special cases of a general integration process based on the invariance of an ordinary differential equation. His idea consisted of studying differential equations via their symmetry groups (San Martin, 1999; Boyer, 2001).

Based on the theory of Lie, Dattoli et al. (1998) developed the rule for the exponential of a first order differential operator. Consider the following first order differential equation

$$
\frac{d F}{d t}=A F
$$

whose formal solution is given by:

$$
F(t)=e^{\int_{0}^{t} A d s} F_{0},
$$

where $F_{0}$ represents the initial condition (Zill and Cullen, 2001).

If $A$ is a differential operator, for example, if

$$
A=-u \frac{\partial}{\partial x}
$$

Eq. (2) becomes:

$$
\frac{\partial F}{\partial t}=-u \frac{\partial F}{\partial x}
$$

From the formal solution given by Eq. (3),

$$
F(x, t)=\left[e^{-\int_{0}^{t} u \frac{\partial}{\partial x} d s}\right\rfloor F_{0}(x) .
$$


If $u$ is considered constant, Eq. (6) becomes:

$$
F(x, t)=\left[e^{-u t \frac{\partial}{\partial x}}\right] F_{0}(x) .
$$

Applying the operator $e^{-u t \frac{\partial}{\partial x}}$ to $F_{0}(x)$, using the Taylor series approximation for the exponential function, i.e.:

$$
e^{-u t \frac{\partial}{\partial x}}=I-u t \frac{\partial}{\partial x}+\frac{1}{2 !}\left(u t \frac{\partial}{\partial x}\right)^{2}-\frac{1}{3 !}\left(u t \frac{\partial}{\partial x}\right)^{3}+\ldots
$$

where I is the identity operator. Equation (8) can be written as:

$$
e^{-u t \frac{\partial}{\partial x}}=\sum_{k=0}^{\infty} \frac{(-1)^{k}}{k !}\left(u t \frac{\partial}{\partial x}\right)^{k} .
$$

Applying Eq. (9) to Eq. (7):

$$
\begin{aligned}
& F(x, t)=F_{0}(x)-u t \frac{\partial}{\partial x} F_{0}(x) \\
& +\frac{1}{2 !}\left(u t \frac{\partial}{\partial x}\right)^{2} F_{0}(x)-\frac{1}{3 !}\left(u t \frac{\partial}{\partial x}\right)^{3} F_{0}(x)+\ldots .
\end{aligned}
$$

This expression can be rewritten as:

$$
F(x, t)=\sum_{k=0}^{\infty} \frac{(-1)^{k}}{k !}\left(u t \frac{\partial}{\partial x}\right)^{k} F_{0}(x) .
$$

It is known that

$$
\begin{aligned}
& F_{0}(x+\Delta x)=F_{0}(x)+\left.\Delta x \frac{\partial F_{0}}{\partial x}\right|_{x}+\left.\frac{(\Delta x)^{2}}{2 !} \frac{\partial^{2} F_{0}}{\partial x^{2}}\right|_{x} \\
& +\left.\frac{(\Delta x)^{3}}{3 !} \frac{\partial^{3} F_{0}}{\partial x^{3}}\right|_{x}+\ldots+\left.\frac{(\Delta x)^{k}}{k !} \frac{\partial^{k} F_{0}}{\partial x^{k}}\right|_{x},
\end{aligned}
$$

if $\Delta x=-u t$ in Eq. (12). Comparing Eqs. (11) and (12):

$$
F(x, t)=F_{0}(x-u t)
$$

Hence, the rule for the exponential of a first order differential operator with constant coefficient (Eq. 14) may be obtained.

$$
\left[e^{c \frac{\partial}{\partial x}}\right] F(x)=F(x+c) .
$$

\section{Rule for Manipulation of the Exponential of a Second Order Differential Operator}

Consider the following second order differential equation with constant coefficient:

$$
\frac{\partial F}{\partial t}=k \frac{\partial^{2} F}{\partial x^{2}}
$$

whose formal solution is given by:

$$
F(x, t)=\left[e^{k \frac{\partial^{2}}{\partial x^{2}}}\right] F(x, 0) .
$$

The use of Taylor series to calculate $F(x, t)$ does not allow obtaining an exponential rule in a straightforward way. In order to develop the rule for the exponential of a second order differential operator, consider the problem:

$$
\left\{\begin{array}{l}
\frac{\partial F}{\partial t}=k \frac{\partial^{2} F}{\partial x^{2}},-\infty<x<\infty, t>0 \\
F(x, 0)=f_{0}(x)
\end{array} .\right.
$$

The application of the Fourier transform to Eq. (17) yields (Reichl, 1980):

$$
\left\{\begin{array}{l}
\frac{\partial \bar{F}(\omega, t)}{\partial t}=-k \omega^{2} \bar{F}(\omega, t) \\
\bar{F}(\omega, 0)=\bar{f}(\omega)
\end{array}\right.
$$

where $\bar{F}(\omega, t)$ denotes the Fourier transform of $F(x, t)$, the Fourier transform of $\frac{\partial^{2}}{\partial x^{2}} F(x, t)$ and $f_{0}(x) \quad$ are $\quad \mathfrak{J}\left\{\frac{\partial^{2}}{\partial x^{2}} F(x, t)\right\}=-\omega^{2} \bar{F}(\omega, t) \quad$ and $\mathfrak{I}\left\{f_{0}(x)\right\}=\bar{f}(\omega)$, respectively. 
The solution of Eq. (18) is:

$$
\bar{F}(\omega, t)=e^{-k \omega^{2} t} \bar{f}(\omega)
$$

The inverse transform gives the formal solution:

$$
F(x, t)=\frac{1}{\sqrt{2 \pi}} \int_{-\infty}^{\infty} e^{i \omega x} e^{-k \omega^{2} t} \bar{f}(\omega) d \omega .
$$

Since

$$
\mathfrak{I}^{-1}\left\{e^{-k \omega^{2} t}\right\}=\frac{1}{\sqrt{2 k t}} e^{-\frac{x^{2}}{4 k t}}
$$

and the convolution theorem yields

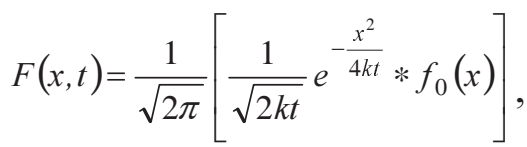

where the symbol $*$ represents the convolution operator, consequently, Eq. (22) gives

$$
F(x, t)=\frac{1}{\sqrt{4 \pi k t}} \int_{-\infty}^{\infty} e^{-\frac{u^{2}}{4 k t}} f_{0}(x-u) d u
$$

where $f_{0}(x)$ stands for the initial state of the system.

Finally, the rule for the exponential of a second order differential operator with constant coefficient can be written as:

$$
\left[e^{k \frac{\partial^{2}}{\partial x^{2}}}\right] f_{0}(x)=\frac{1}{\sqrt{4 \pi k t}} \int_{-\infty}^{\infty} e^{-\frac{u^{2}}{4 k t}} f_{0}(x-u) d u
$$

\section{Rule for the Exponential of First and Second Order Differential Operators with Variable Coefficients}

The rules presented in the last sections cannot be applied when the coefficients of the differential operators vary. Consequently, variable change is applied to the original equation in order to obtain another differential equation with constant coefficients on which the rules can be em- ployed.

In order to obtain the rules for manipulation of exponential of first and second order differential operators with variable coefficient, consider the problem:

$$
\begin{aligned}
& \frac{\partial f}{\partial t}=\frac{\partial}{\partial z}\left(K_{z} \frac{\partial f}{\partial z}\right), \\
& f_{0}(z)=Q \delta\left(z-h_{s}\right) .
\end{aligned}
$$

Equation (25) can be rewritten as

$$
\frac{\partial f}{\partial t}=\frac{\partial K_{z}}{\partial z} \frac{\partial f}{\partial z}+K_{z} \frac{\partial^{2} f}{\partial z^{2}} .
$$

In order to be able to apply the rules, a change of variables is necessary in Eq. (27) which yields a new partial differential equation with constant coefficient:

$$
\frac{\partial f(h, g, t)}{\partial t}=\frac{\partial f(h, g, t)}{\partial g}+\frac{\partial^{2} f(h, g, t)}{\partial h^{2}},
$$

whose formal solution is:

$$
f(h, g, t)=\left[e^{t \frac{\partial}{\partial g}+\frac{\partial^{2}}{\partial h^{2}}}\right] f(h, g, 0),
$$

where the functions $h$ and $g$ are obtained employing the chain rule, i.e.

$$
h=\int \frac{1}{\sqrt{K_{z}}} d z
$$

and

$$
g=2 \int \frac{1}{\left(\frac{d K_{z}}{d z}\right)} d z
$$

The process of developing Eq. (28) in terms of and is shown in the next section.

Applying the rule of manipulation of the second order differential operator given by Eq. (24) to Eq. (29) over variable, yields: 


$$
f(h, g, t)=\left[e^{t \frac{\partial}{\partial g}}\right]\left[\frac{1}{\sqrt{4 \pi t}} \int_{-\infty}^{+\infty} e^{-\frac{\phi^{2}}{4 t}} f(h-\phi, 0) d \phi\right] .
$$

The initial condition given by Eq. (26) can be rewritten in terms of $h$

$$
f_{0}(h)=f_{0}(z(h))=Q \delta\left(z(h)-h_{f}\right),
$$

where $z(h)$ corresponds to the inverse function of $h(z)$.

Thus, Eq. (32) can be rewritten as:

$$
f(h, g, t)=\left[e^{t \frac{\partial}{\partial g}}\right]\left[\frac{1}{\sqrt{4 \pi t}} \int_{-\infty}^{+\infty} e^{-\frac{\phi^{2}}{4 t}} \delta\left(z(h-\phi)-h_{f}\right) d \phi\right]
$$

and the integral in Eq. (34) can be calculated as:

$$
\int_{-\infty}^{+\infty} e^{-\frac{\phi^{2}}{4 t}} \delta\left(z(h-\phi)-h_{f}\right) d \phi=\frac{e^{-\frac{\phi_{1}^{2}(h)}{4 t}}}{\left|p^{\prime}\left(\phi_{1}(h)\right)\right|}+\frac{e^{-\frac{\phi_{2}^{2}(h)}{4 t}}}{\left|p^{\prime}\left(\phi_{2}(h)\right)\right|}
$$

where

$$
p(\phi)=z(h-\phi)-h_{s},
$$

and $\phi_{1}(h)$ and $\phi_{2}(h)$ correspond to the zeros of $p(\phi)$.

Therefore, Eq. (34) is expressed as:

$$
f(h, g, t)=\left[e^{t \frac{\partial}{\partial g}}\right]\left[\frac{1}{\sqrt{4 \pi t}}\left(\frac{e^{-\frac{\phi_{1}^{2}(h)}{4 t}}}{\mid p^{\prime}\left(\phi_{1}\right)}+\frac{e^{-\frac{\phi_{2}^{2}(h)}{4 t}}}{\left|p^{\prime}\left(\phi_{2}\right)\right|}\right)\right]
$$

since $h$ can be indirectly written in terms of $g$,

$$
\begin{aligned}
& f(h, g, t)=\left[e^{t \frac{\partial}{\partial g}}\right] \\
& {\left[\frac{1}{\sqrt{4 \pi t}} \frac{e^{-\frac{\phi_{1}^{2}(h(z(g)))}{4 t}}}{\left|p^{\prime}\left(\phi_{1}(h(z(g)))\right)\right|}+\frac{e^{-\frac{\phi_{2}^{2}(h(z(g)))}{4 t}}}{\mid p^{\prime}\left(\phi_{2}(h(z(g)))\right)}\right] .}
\end{aligned}
$$

Applying the rule for the exponential of a first order differential operator given by Eq. (14) over variable $g$,

$$
\begin{aligned}
& f(h, g, t)=\frac{1}{\sqrt{4 \pi t}} \cdot \\
& {\left[\frac{e^{-\frac{\phi_{1}^{2}(h(z(g-t)))}{4 t}}}{\mid p^{\prime}\left(\phi_{1}(h(z(g-t)))\right)}+\frac{e^{-\frac{\phi_{2}^{2}(h(z(g-t)))}{4 t}}}{\mid p^{\prime}\left(\phi_{2}(h(z(g-t)))\right)}\right]}
\end{aligned}
$$

As $g$ can be expressed in terms of $z$, the solution of Eq. (25) may be expressed in function of $z$ and $t$, i.e.,

$$
f(z, t)=\frac{1}{\sqrt{4 \pi t}} .
$$$$
\left[\frac{e^{-\frac{\phi_{1}^{2}(h(z(g(z)-t)))}{4 t}}}{\left|p^{\prime}\left(\phi_{1}(h(z(g(z)-t)))\right)\right|}+\frac{e^{-\frac{\phi_{2}^{2}(h(z(g(z)-t)))}{4 t}}}{\mid p^{\prime}\left(\phi_{2}(h(z(g(z)-t)))\right)}\right] .
$$

\section{Consequently:}

$$
\begin{gathered}
f(z, t)=\left[e^{\frac{\partial K_{z} \partial c}{\partial z} \frac{\partial c}{\partial z}+K_{z} \frac{\partial^{2} c}{\partial z^{2}}}\right] f(z, 0)=\frac{1}{\sqrt{4 \pi t}} \cdot \\
{\left[\frac{e^{-\frac{\phi_{1}^{2}(h(z(g(z)-t)))}{4 t}}}{\mid p^{\prime}\left(\phi_{1}(h(z(g(z)-t)))\right)}+\frac{e^{-\frac{\phi_{2}^{2}(h(z(g(z)-t)))}{4 t}}}{\left|p^{\prime}\left(\phi_{2}(h(z(g(z)-t)))\right)\right|}\right]}
\end{gathered}
$$

\section{Developing the Expressions for the Functions $g$ and $h$}

From

$$
K_{z} \frac{\partial^{2}}{\partial z^{2}}+\left(\frac{\partial K_{z}}{\partial z}\right) \frac{\partial}{\partial z}=\frac{\partial^{2}}{\partial h^{2}}+\frac{\partial}{\partial g}
$$

and employing the chain rule, the following expressions are readily obtained:

$$
\frac{\partial}{\partial h}=\frac{1}{\left(\frac{\partial h}{\partial z}\right)} \frac{\partial}{\partial z}
$$


and

$$
\frac{\partial}{\partial g}=\frac{1}{\left(\frac{\partial g}{\partial z}\right)} \frac{\partial}{\partial z}
$$

Differentiating Eq. (43) with respect to $h$,

$$
\frac{\partial}{\partial h}\left(\frac{\partial}{\partial h}\right)=\frac{\partial}{\partial h}\left[\frac{1}{\left(\frac{\partial h}{\partial z}\right)} \frac{\partial}{\partial z}\right],
$$

it yields

$$
\frac{\partial^{2}}{\partial h^{2}}=-\frac{1}{\left(\frac{\partial h}{\partial z}\right)^{3}} \frac{\partial^{2} h}{\partial z^{2}} \frac{\partial}{\partial z}+\frac{1}{\left(\frac{\partial h}{\partial z}\right)^{2}} \frac{\partial^{2}}{\partial z^{2}} .
$$

The substitution of Eq. (44) and Eq. (46) in Eq. (42), yields

$$
\begin{aligned}
& K_{z} \frac{\partial^{2}}{\partial z^{2}}+\left(\frac{\partial K_{z}}{\partial z}\right) \frac{\partial}{\partial z} \\
& =\frac{1}{\left(\frac{\partial h}{\partial z}\right)^{2}} \frac{\partial^{2}}{\partial z^{2}}+\left\{\frac{1}{\left(\frac{\partial g}{\partial z}\right)}-\frac{1}{\left(\frac{\partial h}{\partial z}\right)^{3}} \frac{\partial^{2} h}{\partial z^{2}}\right\} \frac{\partial}{\partial z},
\end{aligned}
$$

where the coefficient of the first and second order differential operators are the same on both sides of Eq. (47), thus

$$
K_{z}=\frac{1}{\left(\frac{\partial h}{\partial z}\right)^{2}}
$$

and

$$
\frac{\partial K_{z}}{\partial z}=\frac{1}{\left(\frac{\partial g}{\partial z}\right)}-\frac{1}{\left(\frac{\partial h}{\partial z}\right)^{3}} \frac{\partial^{2} h}{\partial z^{2}} .
$$

Therefore,

$$
h=\int \frac{1}{\sqrt{K_{z}}} d z
$$

$$
g=2 \int \frac{1}{\left(\frac{d K_{z}}{d z}\right)} d z
$$

Solution of the Three-dimensional Advection Diffusion Equation in a Cartesian Coordinates System

The problem of pollutant dispersion on the atmosphere can be mathematically described by:

$$
\begin{aligned}
& \frac{\partial c}{\partial t}+u \frac{\partial c}{\partial x}+v \frac{\partial c}{\partial y}+w \frac{\partial c}{\partial z} \\
& =\frac{\partial}{\partial z}\left(K_{z} \frac{\partial c}{\partial z}\right)+\frac{\partial}{\partial x}\left(K_{x} \frac{\partial c}{\partial x}\right)+\frac{\partial}{\partial y}\left(K_{y} \frac{\partial c}{\partial y}\right)
\end{aligned}
$$

subjected to the initial condition

$$
c(x, y, z, 0)=c_{0}(x, y, z)=Q r(x, y) \delta\left(z-h_{s}\right)
$$

where $c$ is the pollutant concentration, $t$ is the period of time since the pollutant emission, $K_{x}, K_{y}$ and $K_{z}$ are the longitudinal, transverse and vertical diffusion coefficients, respectively, $h_{s}$ is the height of the source, $u, v$ and $w$ correspond to the mean wind components in the $x, y$ and $z$ directions, respectively, and $S$ is the source term.

Coefficients $K_{x}$ and $K_{y}$ as well as the wind speed in the $x$ direction, assumed as dominant, are considered constant. Thus, Eq. (52) can be rewritten as

$$
\frac{\partial c}{\partial t}+u \frac{\partial c}{\partial x}=\frac{\partial}{\partial z}\left(K_{z} \frac{\partial c}{\partial z}\right)+K_{x} \frac{\partial^{2} c}{\partial x^{2}}+K_{y} \frac{\partial^{2} c}{\partial y^{2}}
$$

whose formal solution is:

$$
\begin{aligned}
& c(x, y, z, t)= \\
& {\left[e^{-t u \frac{\partial}{\partial x}+K_{x} \frac{\partial^{2}}{\partial x^{2}}+K_{y}} \frac{\partial^{2}}{\partial y^{2}}+K_{z} \frac{\partial^{2}}{\partial z^{2}}+t\left(\frac{\partial K_{z}}{\partial z}\right) \frac{\partial}{\partial z}\right] c(x, y, z, 0)}
\end{aligned}
$$

or

$$
\begin{aligned}
& c(x, y, z, t)=\left[e^{-t u \frac{\partial}{\partial x}}\right]\left[e^{\left.K_{x} \frac{\partial^{2}}{\partial x^{2}}\right] .}\right. \\
& {\left[e^{\left.K_{y} \frac{\partial^{2}}{\partial y^{2}}\right]}\right] e^{\left.K_{z} \frac{\partial^{2}}{\partial z^{2}}+t\left(\frac{\partial K_{z}}{\partial z}\right) \frac{\partial}{\partial z}\right] c(x, y, z, 0) .}}
\end{aligned}
$$


In order to obtain the solution of Eq. (54), the rules for manipulation of the exponential of the differential operators are employed. Initially, the rule given by Eq. (41) is applied to Eq. (56) over z, producing Eq. (57)

$$
A(z)=\frac{e^{-\frac{\phi_{1}^{2}(h(z(g(z)-t)))}{4 t}}}{\mid p^{\prime}\left(\phi_{1}(h(z(g(z)-t)))\right)}+\frac{e^{-\frac{\phi_{2}^{2}(h(z(g(z)-t)))}{4 t}}}{\mid p^{\prime}\left(\phi_{2}(h(z(g(z)-t)))\right)},
$$

and

$$
c(x, y, z, t)=\left[e^{-t u \frac{\partial}{\partial x}}\right]\left[e^{K_{x} \frac{\partial^{2}}{\partial x^{2}}}\right]\left[e^{K_{y} \frac{\partial^{2}}{\partial y^{2}}}\right]\left[\frac{Q r(x, y)}{\sqrt{4 \pi t}}(A(z))\right] .
$$

Applying the rule for manipulation of the exponential of a second order differential operator with constant coefficient given by Eq. (24) over $y$ to Eq. (58) yields:

$$
\begin{aligned}
& c(x, y, z, t) \\
& =\left[e^{-t u \frac{\partial}{\partial x}}\right]\left[e^{K_{x} \frac{\partial^{2}}{\partial x^{2}}}\right]\left[\int_{-\infty}^{+\infty} \frac{Q e^{-\frac{\beta^{2}}{4 K_{y}}} r(x, y-\beta)}{\sqrt{4 \pi t}}(A(z)) d \beta\right]
\end{aligned}
$$

Employing the rule for manipulation of the exponential of a second order differential operator with constant coefficient given by Eq. (24) over $x$ to Eq. (59) yields:

$$
\begin{aligned}
& c(x, y, z, t)=\left[e^{-t u \frac{\partial}{\partial x}}\right] \\
& {\left[\frac{A(z)}{\sqrt{4 \pi t}} \int_{-\infty}^{+\infty} \int_{-\infty}^{+\infty} Q e^{-\frac{\gamma^{2}}{4 t K_{x}}} e^{-\frac{\beta^{2}}{4 t K_{y}}} r(x-\gamma, y-\beta) d \beta d \gamma\right] .}
\end{aligned}
$$

Finally, applying the rule for manipulation of a exponential of a first order differential operator given by Eq. (14) over $x$ to Eq. (60) yields:

$$
\begin{aligned}
& c(x, y, z, t)=\frac{A(z)}{\sqrt{4 \pi t}} \cdot \\
& \int_{-\infty-\infty}^{+\infty+\infty} Q e^{-\frac{\gamma^{2}}{4 t K_{x}}} e^{-\frac{\beta^{2}}{4 t K_{y}}} r(x-\gamma-u t, y-\beta) d \beta d \gamma
\end{aligned}
$$

which corresponds to the solution of Eq. (54) where

$$
r(x, y)=\delta\left(x-x_{s}\right) \delta\left(y-y_{s}\right)
$$

and $x_{s}$ and $y_{s}$ are the coordinates of the location of the source.

Although the process of obtaining the solution seems costly, it is performed just once. Also, it is important to emphasize that the formulation is presented in a generic form, without specifying the parameters involved in the application. When applied to practical examples, the process of obtaining the solution becomes straightforward.

The solution given by Eq. (61) can be used to simulate the pollutant dispersion for different models of the dispersion coefficient. Taking into account that the result corresponds to Gaussian functions in $x$ and $y$ directions, in this paper, the rule for manipulation of the exponential of the second order differential operator for $\mathrm{z}$ component is tested using the diffusion coefficient $K_{z}$ proposed by Degrazia and Moraes (1992), which allows the comparison with the results obtained by Moura (1995). In order to achieve that, the following problem is solved:

$$
\begin{aligned}
& \frac{\partial c}{\partial t}=\frac{\partial}{\partial z}\left(K_{z} \frac{\partial c}{\partial z}\right), \\
& c_{0}(z)=Q \delta\left(z-h_{s}\right),
\end{aligned}
$$

where

$$
K_{z}=\frac{0.33(1-z / h)^{\alpha_{1} / 2}(z / h)}{1+3.7(z / h)(h / \Lambda)} u_{*} h
$$

where $h_{s}$ corresponds to the source's height, $Q=400 \mathrm{~g} / \mathrm{m}^{2}$ is the source's intensity, $h=400 \mathrm{~m}$ represents the boundary layer's height. $\alpha_{1}=2$, $\alpha_{2}=3$ and $u_{*}=0.31 \mathrm{~m} / \mathrm{s}$, the friction velocity, were obtained from Minnesota experiment. Also, $\frac{\Lambda}{L}=(1-(z / h))^{\left[3 / 2 \alpha_{1}-\alpha_{2}\right]}, \Lambda$ is the local Monin-Obukov length and $L=116 \mathrm{~m}$ is the Monin-Obukov length obtained from Minnesota experiment. (Moura, 1995)

\section{RESULTS}

In this paper, turbulent transport in a stable boundary layer was analyzed. Figure 1 shows the time evolution of the pollutant concentration in a $12.5 \mathrm{~m}$-high point, located $80 \mathrm{~m}$ far from the source. 
The results obtained by the formulation presented in this paper agree with the ones published by Moura (1995) as may be confirmed in Fig. 1. The mean square deviation is approximately $6 \%$.

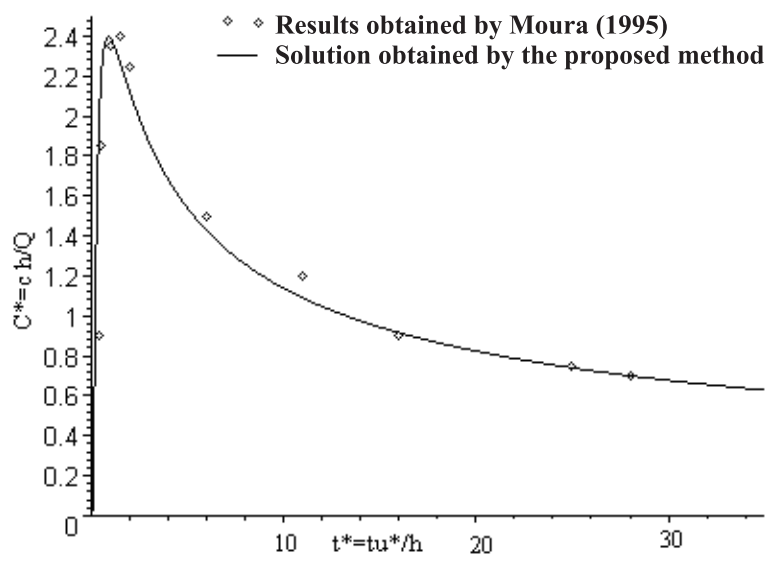

Figure 1. Time evolution of the pollutant concentration found at $12.5 \mathrm{~m}$ of height, $80 \mathrm{~m}$ far from the source

\section{CONCLUSIONS}

The solution presented is analytical and can be easily used for post-processing in software of symbolic compoutation using microcomputers. Besides, it is advantageous when compared with other analytical solutions that apply Laplace transform (Moura, 1995; Moreira, 2000), since there is no need of discretizing the domain. The processing time is very low (20 s in a Pentium IV, 1.7 Ghz). Maple VTM was used to obtain the analytical solution in real time, requiring a small amount of memory to perform the task involved in obtaining the exact solution. The application of the rules increased the processing velocity, reduced the amount of memory needed to perform the post-processing tasks and abolished the need of domain discretization.

\section{REFERENCES}

Boyer, C., 2001. História da Matemática, Edgard Blücher. (in Portuguese)

Chrysikopoulous, C., Hildemann, L., and Roberts, P., 1992, A 3-dimensional Steady-state Atmospheric Dispersion Deposition Model for Emissions from a Ground Area Source, Atmospheric Environment - Part A, Vol. 26A, No. 5, pp. 747-757.

Dattoli, G., Giannesi, L., Quatromini, M., and Torre, A., 1998, Exponential Operators, Operational Rules and Evolution Problems, Il Nuovo Cimento, Vol. 113B, No. 6, pp. 699-710.
Degrazia, G., and Moraes, O, 1992, A Model for Eddy Diffusivity in a Stable Boundary Layer, Boundary-Layer Meteorology, Vol. 58, pp. 205-214.

Koch, W., 1989, A Solution of the Twodimensional Atmospheric Diffusion Equation with Height-dependent Diffusion Coefficients Including Ground Level Absorption, Atmospheric Environment, Vol. 23, No. 8, pp. 1729-1732.

Moreira, D., 1996, Comparação entre um Modelo Gaussiano Atualizado e a Equação de Difusão no Cálculo da Concentração Superficial de Poluentes na Camada Limite Convectiva: o Caso de Fontes Pontuais Contínuas Elevadas, MSc Dissertation, UFSM, Santa Maria, RS. (in Portuguese)

Moreira, D., 2000, Modelo Euleriano Semianalítico de Difusão Turbulenta de Contaminante, Doctoral Thesis, UFRGS, Porto Alegre, RS. (in Portuguese)

Moura, A., 1995, Solução Analítica para Dispersão Vertical Turbulenta em uma Camada Limite Estável, MSc Dissertation, UFRGS, Porto Alegre, RS. (in Portuguese)

Moura, A., 1999, Modelos Multidimensionais Analíticos de Dispersão de Contaminantes na Atmosfera: Coeficientes de Difusão Dependentes da Distância da Fonte, Doctoral Thesis, UFRGS, Porto Alegre, RS. (in Portuguese)

Nieuwstadt, F., 1980, An Analytic Solution of the Time-dependent, One-dimensional Diffusion Equation in the Atmospheric Boundary Layer, Atmospheric Environment, Vol. 14, pp. 1361-1364.

Pires, C., 1996, Um Estudo Analítico de Dispersão de Contaminantes Abandonados por Fontes Áreas em uma Camada Limite Convectiva, MSc Dissertation, UFRGS, Porto Alegre, RS. (in Portuguese)

Reichl, L., 1980, A Modern Course in Statistical Physics, Edward Arnold, London.

San Martin, L., 1999, Álgebras de Lie, Unicamp. (in portuguese)

Zabadal, J., Poffal, C., and Bogado, S., 2004. Simulation of Two Dimensional Viscous Flows Using Lie Algebra, In: XXV Iberian Latin American Congress on Computational Methods Engineering, Pernambuco, Brazil.

Zabadal, J., Vilhena, M., and Segatto, C., 2001, The Total Photon Cross-section Formulation for a Wide Range of Energy, Il Nuovo Cimento, Vol. 116B, No. 2, pp. 183-190.

Zill, D., and Cullen, M., 2001, Equações Diferenciais, Makron Books. (in Portuguese) Zwillinger, D., 1997, Handbook of Differential Equations, Academic Press. 